\title{
The Two-particle Picture and Electronic Structure Calculations
}

\author{
A Gonis \\ T C Schulthess \\ P E A Turchi
}

This paper was prepared for submittal to the

International Workshop on Election Correlations and Materials Ptoperties Crete, Greece

June 28, 1998 through July 3, 1998

June 24, 1998

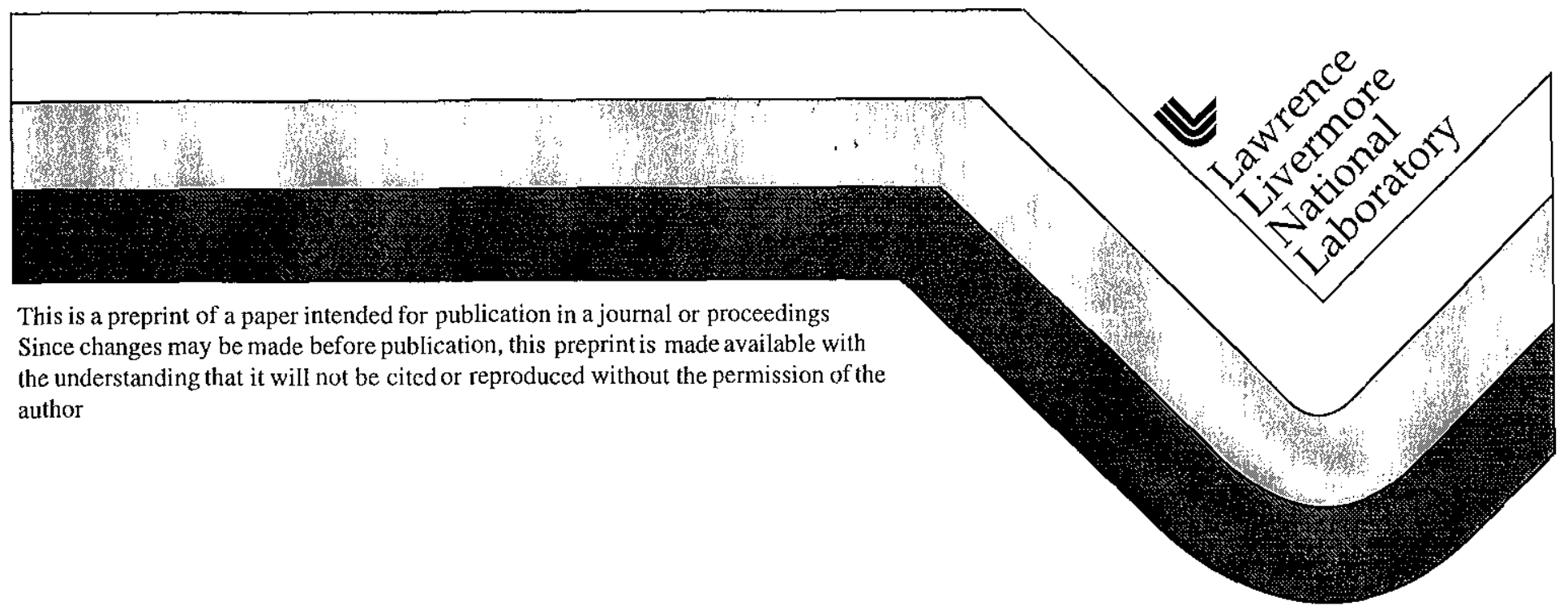




\section{DISCLAIMER}

This document was prepared as an account of work sponsored by an agency of the United States Government Neither the United States Government nor the University of California nor any of their employees, makes any warranty, express or implied, or assumes any legal liability or responsibility for the accuracy, completeness, or usefulness of any information, apparatus, product, or process disclosed, or represents that its use would not infringe privately owned rights Reference herein to any specific commercial product, process, or service by trade name, trademark, manufacturer, or otherwise, does not necessarily constitute or imply its endorsement, recommendation, or favoring by the United States Government or the University of California. The views and opinions of authors expressed herein do not necessarily state or reflect those of the United States Government or the University of California, and shall not be used for advertising or product endorsement purposes 


\title{
The two-particle picture and electronic structure calculations
}

\author{
A. Gonis ${ }^{1}$, T C. Schulthess ${ }^{2}$, and P. E. A. Turchi ${ }^{1}$ \\ 1 Department of Chemistry and Materials Science, L-268 \\ Lawrence Livermore National Laboratory, Livermore, CA 94550 \\ 2 Metals and Ceramics Division, Oak Ridge National Laboratory \\ Oak Ridge, TN 37831
}

We derive exact formal expressions for the self-energy, $\Sigma^{(n)}$, describing the interaction of $n$ particles with one another and with the rest of the particles in an interacting quantum $N$-particle system In contrast to traditional treatments, in which the single-particle self-energy is built out of interactions of a particle with the rest of the system, here a general $n$-particle quantity, $\Sigma^{(n)}$, is obtained in a straightforward fashion by integrating the exact $N$-particle Green function, $G^{(N)}$, over the coordinates of $N-n$ particles and inverting An alternative expression, based on the canonical many-body equation of motion for the Green function is also discussed and compared with that derived through the integration process. The methodology is developed with respect to two-particle states, with the two-particle Green function being the central quantity from which the single-particle self-energy and Green function are derived It is suggested that the two-particle Green function be calculated directly in six-dimensional space in a two-particle generalization of density functional theory and the corresponding local density approximation. Methods for the calculation of the single-particle, $n=1$, self-energy and effective single-particle t-matrix are discussed, and the methodology is illustrated by means of calculations on a model system

\section{INTRODUCTION}

The consept of self-energy figures prominently in the studies of interacting quantum many-particle systems This quantity is generally defined in terms of the energy change that accompanies the addition of a single particle to the interacting system and incorporates exactly the effects of correlation and the statistics, Fermi-Dirac or Bose-Einstein The single-particle self-energy is non-local, generally complex, and also energy-dependent Its evaluation is ususally associated with various approximate, perturbative or decoupling, schemes as described[1,2] in the literature.

In this paper, we introduce a point of view which differs from canonical treat- 
ments of interacting quantum systems. Instead of building the self-energy from individual contributions to the interaction of a particle with the rest of the system, e $\mathrm{g}$, summations of selected diagrams, we treat the entire system of $N$ interacting threedimensional particles as a single particle in a configurational space of $3 N$ dimensions. In the non-relativistic limit considered here, the external potentials acting on the particles as well as their mutual interactions appear as a time-independent externally applied field Consequently, the Green function of the system can be expressed in the familiar form obtained in the traditional single-particle picture It is shown that from a knowledge of the Green function describing $n$ interacting particles (in an $N$-particle system, with $N$ being either finite or infinite) Green functions for $m$ interacting particles, $m<n$, can be obtained directly Inverting the resulting $m$-particle Green function leads directly to the self-enrgy of the $m$-particle unit, extending the concept of self-energy to an $m$-particle quantity. It is also shown that the solution of the equation of motion for the many-particle Green function leads to an alternative form for this quantity The two forms are compared and contrasted in the final discussion Furthermore, the formulation in terms of $t$-matrices for both kinds of Green functions given below provides a particularly flexible framework for the development of approximate computational schemes for evaluating Green functions and the corresponding self-energies

Another aim of the discussion is to indicate certain formal connections between canonical many-body theory and band theory, the methodology used in the study of the elctronic structure of matter, and properties related to it Band theory is currently being implemented within the context of the local (spin) density approximation (L(S)DA) of density functional theory (DFT)[3]. The DFT-LDA approach has been very successful in the treatment of metallic solids[4], but is often criticized for its approximate treatment of correlation effects It is known to provide a poor desription of the gap in the single-particle spectrum of semiconductors and insulators, either understimating the gap, or missing it altogether, predicting metallic behavior It also fails to describe the volume collapse that accompanies increased pressure in many elemental solids of the Lanthanide and Actinide series. Possibly most important is the lack of a well-defined procedure for extending the DFT-LDA approach to alleviate these shortcomings

It is shown below that it is possible to view DFT-LDA and canonical manybody theory from a single perspective as two parts of a coherent whole As such, it affords a unique, formally exact procedure for improvement, and provides a single parameter, namely the number of particles treated explicitly, as a parameter governing the convergence to exact results.

The following discussion is confined to the non-relativistic limit so that the inter- 
action between two particles is taken to be instantaneous, $U\left(\mathbf{r} t, \mathbf{r}^{\prime} t^{\prime}\right)=U\left(\mathbf{r}, \mathbf{r}^{\prime}\right) \delta\left(t-t^{\prime}\right)$ It is, however, general enough to accommodate a diverse class of potentials that include the nuclear forces in an atom or a molecule, as well as in extended systems such as a solid

\section{THE GREEN FUNCTION AND SELF-ENERGY}

We confine our attention to an interacting, $N$-particle system described by the Hamiltonian,

$$
H^{(N)}=\sum_{i=1}^{N} h\left(x_{i}\right)+\frac{1}{2} \sum_{i \neq j} U\left(x_{i}-x_{j}\right)
$$

where $h\left(x_{i}\right)$ is the Hamiltonian describing a single particle and includes the kinetic energy and any external potential acting on the system, while $U\left(x_{i}-x_{j}\right)$ denotes the interparticle interaction Here, $x_{n}=\left(\mathrm{r}_{n} t_{n}\right)$ is a combined space-time coordinate for the $n$th particle, and $\mathrm{d}^{4} x_{n}=\mathrm{d}^{3} r_{n} \mathrm{~d} t_{n}$. (This coordinate can also incorporate the spin variable but this will not be made explicit in the discussion.)

We begin with the familiar $[5,6]$ equation of motion for the many-particle Green function Treating explicitly the case of fermions, we have

$$
\begin{aligned}
& {\left[\mathrm{i} \hbar \frac{\partial}{\partial t_{1}}+\frac{\hbar^{2}}{2 m} \nabla_{1}^{2}\right] G^{(n)}\left(x_{1}, x_{2}, \quad, x_{n} ; x_{1}^{\prime}, x_{2}^{\prime}, \quad, x_{n}^{\prime}\right) } \\
+ & \mathrm{i} \hbar \int \mathrm{d}^{4} x_{n+1} U\left(x_{1}-x_{n+1}\right) \\
\times & G^{(n+1)}\left(x_{1}, \cdot, x_{n+1} ; x_{1}^{\prime}, \cdots, x_{n+1}^{\prime}\right) \\
= & \sum_{i=1}^{n} \delta^{(4)}\left(x_{1}-x_{i}^{\prime}\right)(-1)^{i-1} \\
\times & G^{(n-1)}\left(x_{2}, \quad, x_{n}, x_{1}^{\prime}, \cdot, x_{i-1}^{\prime}, x_{i+1}^{\prime}, \quad, x_{n}^{\prime}\right) .
\end{aligned}
$$

Also, any external "single-particle" potential, such as that due to the nuclei in a molecule or a solid, can be incorporated explicitly inside the brackets in the last expression For the sake of clarity of presentation, we continue to treat explicitly the uniform case, $h(x)=\nabla_{\mathbf{r}}^{2}$

It is seen that in general the equation for the $n$-particle Green function contains both the $n+1$ - and the $n-1$-particle Green functions Defining the free-particle propagator, $G_{0}=\left[\mathrm{i} \hbar \frac{\partial}{\partial t}+\frac{\hbar^{2}}{2 m} \nabla^{2}\right]^{-1}$, setting all $t_{i}=t$ and $t_{i}^{\prime}=t^{\prime}$, (describing the probability amplitude of creating all particles at time $t^{\prime}$ and annihilating them at time $t$ ), and Fourier transforming with respect to $t^{\prime}-t$ into the energy domain, we obtain, 


$$
\begin{aligned}
& G^{(N)}\left(r_{1}, r_{2}, \cdot, r_{N} ; r_{1}^{\prime}, r_{2}^{\prime}, \quad, r_{N}^{\prime}, E\right) \\
= & \sum_{i=1}^{N}(-1)^{i-1} \int \mathrm{d} \omega G_{0}\left(r_{1}, r_{i}^{\prime}, \omega\right) \\
\times & G^{(N-1)}\left(r_{2}, \quad, r_{N}, r_{1}^{\prime}, \cdot, r_{i-1}^{\prime}, r_{i+1}^{\prime}, \quad, r_{N}^{\prime}, E-\omega\right)
\end{aligned}
$$

This expression is properly antisymmetric with respect to interchange of fermion coordinates It describes the probability amplitude that the system of $N$ particles propagates from a state with position $\mathbf{r}_{1}^{\prime}, \cdot, \mathbf{r}_{N}^{\prime}$ to the state with positions $\mathbf{r}_{1}, \quad, \mathbf{r}_{N}$

Let us now return to $\mathrm{Eq}(3)$ We use the definition of the Green function as a probability amplitude for creating (destroying) particles to sum (or integrate) over the coordinate of a given particle, say particle 1, and interpret the result as the Green function for $N-1$ particles moving in the presence of a single particle Assuming that $h^{(1)}$, the Hamiltonian associated with a single, non interacting particle, is translationally invariant (or uniform), this summation yields the $\mathrm{k}=0$ element of the single-particle Green function, $G_{0}$. Since this is given by the general expression $G_{0}(\mathbf{k}=0 ; z)=\left[z-h^{(1)}(\mathrm{k}=0)\right]^{-1}$, the remaining integration yields a $N-1$-order Green function which can be written in the form,

$$
G^{(N-1)}(z)=\left[z-\Sigma^{(N-1)}\right]^{-1}
$$

Here, the self-energy $\Sigma^{(N-1)}$ is non-local in the coordinates of the $N-1$ particles, complex, and energy dependent As defined here, it also contains a shift by the energy at $\mathbf{k}=0$ of a uniform (or periodic) single-particle system, but that shift will not be shown explicitly. Thus, summation over the coordinates of the Green function elements assocrated with a single particle in $G^{(N)}$ yields a Green function, $G^{(N-1)}$, describing the motion of $N-1$ in the $N$-particle system Let us denote this reduced-order quantity by a hat symbol, (because it contains a shifted self-energy), and refer as downfolding to the process of summing over the coordinates (or Green function elements) associated with given particles in the system In operator space, the previous discussion can be summarized in the form,

$$
\begin{aligned}
& \hat{G}^{(N-1)}\left(\imath_{2}, \quad, \imath_{N} ; i_{2}^{\prime}, \quad, \imath_{N}^{\prime}, z\right) \\
= & \frac{1}{\Omega} \sum_{i_{1}, i_{1}^{\prime}} G^{(N)}\left(\imath_{1}, \imath_{2}, \cdot, \imath_{N}, \imath_{i}^{\prime}, \imath_{2}^{\prime}, \quad, \imath_{N}^{\prime} ; z\right),
\end{aligned}
$$

where in the coordinate representation the summations are to be interpreted as integrals over the volume, $\Omega$, of the system More generally, it follows from this discussion that a reduced Green function for $n$ particles can be obtained by downfolding the exact $N$-particle Green function, 


$$
\begin{aligned}
\hat{G}^{(n)}(z) & =\frac{1}{\Omega^{N-n}} \\
& \times \sum_{\substack{i_{1}, i_{2}, i_{1}^{\prime}, i_{2}^{\prime}, i_{N-n}^{\prime}, i_{N-n}^{\prime}}} G^{(N)}\left(\imath_{1}, \imath_{2}, \quad, \imath_{N} ; \imath_{i}^{\prime}, \imath_{2}^{\prime}, \cdots, \imath_{N}^{\prime}, z\right),
\end{aligned}
$$

over the coordinates of $N-n$ particles Finally, we see that the self-energy for a system of $n$ particles is given by the general expression,

$$
\Sigma^{(n)}\left(\imath_{1}, \imath_{2}, \quad, \imath_{n}, \imath_{1}^{\prime}, \imath_{2}^{\prime}, \quad, \imath_{n}^{\prime}, z\right)=z-\left[\hat{G}^{(n)}\right]^{-1}
$$

The single-particle Gieen function and self-energy can now be obtained by setting $n=1$, and downfolding $G^{(N)}$ over the coordinates of $N-1$ particles

\section{THE T-MATRIX}

It is starightforward to cast the formal developments presented above in terms of an effective t-matrix Here, the t-matrix of the exact $N$-particle system is defined by the expression,

$$
G^{(N)}=G_{0}^{(N)}\left[1+T^{(N)} G_{0}^{(N)}\right]
$$

which in the space of $N$ particles can be developed in the form of a Born series,

$$
T^{(N)}=U^{(N)}+U^{(N)} G_{0}^{(N)} U^{(N)}+\cdots .
$$

Here, $U^{(N)}$ contains the mutual interactions of all $N$ particles and, in $N$-particle space, appears as an external potential. The quantities $G_{0}^{(N)}$ describe the force-free propagation of the entire system of particles It is straightforward to show that the downfolding procedure described above leads to a reduced-order Green function which can be expressed in terms of a t-matrix For $n<N$, we have

$$
G^{(n)}=\hat{G}_{0}^{(n)}+\hat{G}_{0}^{(n)} \hat{T}^{(n)} \hat{G}_{0}^{(n)},
$$

where $\hat{T}^{(n)}$ is obtained through a downfolding process over the coordinates of $N-n$ particles,

$$
\begin{aligned}
\hat{T}^{(n)}(z) & =\frac{1}{\Omega^{N-n}} \\
& \times \sum_{\substack{i_{1}, i_{2}, i_{1}^{\prime}, i_{2}^{\prime},, i_{N-n}^{\prime} \\
, i_{N-n}^{\prime}}} T^{(N)}\left(\imath_{1}, \imath_{2}, \quad, \imath_{N}, \imath_{i}^{\prime}, i_{2}^{\prime}, \quad, \imath_{N}^{\prime}, z\right) .
\end{aligned}
$$

Also, the self-energy in $n$-particle space is given by the well-known relation, 


$$
\Sigma^{(n)}=\hat{T}^{(n)}\left[1+\hat{\bar{G}}_{0}^{(n)} \hat{T}^{(n)}\right]^{-1}
$$

It follows from the derivation, that the self-energy obtained through this procedure is identical to that obtained through the Green function, Eq (7) However, the evaluation of the t-matrix is considerably easier than that of the Green function because the t-matrix is non vanishing when all of its arguments lie in the potential region Thus, the downfolding process extends only over a finite region, namely where the potential is acting, rather than over all space as required when dealing with the Green function itself

\section{NUMERICAL RESULTS}

The previous discussion can be illustrated by means of a "small" one-dimensional system described by a Hubbard-like model Hamiltonian,

$$
H=\sum_{i} \epsilon_{i}|\imath\rangle\left\langle\imath\left|+\sum_{i \neq j} t_{i j}\right| \imath\right\rangle\langle j|+\sum_{i} U_{i} n_{i \uparrow} n_{i \downarrow}
$$

This Hamiltonian is taken to describe a system of four electrons with total spin zero arranged on the four sites of a closed linear ring characterized by site energies $\epsilon_{i}=0.0$ (uniform potential) The quantities $|i\rangle$ denote the state associated with site $\imath, t_{i j}$ is a trănsfer integral between sites $\imath$ and $j$, and $U_{i}$ is an on-site Coulomb repulsion term that is associated with two electrons of opposite spin occupying the same site. The quantities $n_{i \sigma}$ are the number operators for an electron on site $\imath$ with spin $\sigma$.

The Hamiltonians in two-particle space and in four-particle space can be defined from that in Eq.(13) on the tensor product of the corresponding single-particle spaces Let $I_{\left\{n_{1}, n_{2}, n_{m}\right\}}^{(m)}$ denote the identity operator in $m$-particle space of the set of particles $\left\{n_{1}, n_{2}, n_{m}\right\}$, and let $H_{\left\{n_{1}, n_{2}, n_{m}\right\}}^{(m)}$ be the corresponding Hamiltonian. With $H_{1}^{(1)}$ given by Eq (13), we have,

$$
H_{1,2}^{(2)}=H_{1}^{(1)} \otimes I_{2}^{(1)}+H_{2}^{(1)} \otimes I_{2}^{(1)}+U^{(12)},
$$

where $U^{(12)}$ denotes the operator describing the interaction of two particles in twoparticle space The Hamiltonian of the four-particle system can be constructed in a straightforward application of combining tensor products of spaces, and adding the corresponding interparticle interaction

Now, the a four-particle Green function for this system can be obtained by inverting the four-dimensional structure formed by the orthogonal combination of four four-sited rings in the manner just indicated. This space contains 256 configurations describing all possible arrangements of four electrons, two with spin up and two with spin down, on the four sites of the ring These configurations are consistent with the 
Pauli exclusion principle, in that an "infinite" $\left(\approx 10^{7}\right)$ potential was associated with any configuration in which two or more electrons of the same spin occupy the same site In the calculations reported here, we restrict the range of electron hopping, $t$, to nearest neighbors, and set $U / t=10.0$ Also, for ease of illustration, the spectra are exhibited for values of the energy that contain an imaginary part, $\Im E=t / 4$

An approximate evaluation of the Green function and the self-energy can be made by considering separately the sixteen different two-particle Green functions obtained for each configuration of the other two particles in the system There are sixteen such configurations whose mutual interactions are neglected Thus, each two-particle unit is described by an effective Hamiltonian which corresponds to each possible configuration of the remaining two particles in the system

The solid line in the upper panel of Fig 1 shows the exact single-particle spectrum obtained from downfolding the exact four-particle Green function The dotted line corresponds to the approximate evaluation just described The lower two panels of the figure shows the real and imaginary parts of the corresponding site-diagonal element of the self-energy. It is seen that the approximate spectra reproduce rather well the main features of the exact ones, in particular the impurity-like peak at about $\Re E=U$ The exact spectra lead to a narrower distribution of eigenvalues (band in solids) since they take proper account of the configuration interactions Because of the Pauli principle, which prevents two particles of the same spin from occupying the same site, the available configuration space of the system is reduced leading to a narrowing of the "band" at the four-particle level as well as at the single-particle level The difference in structure inside the main part of the spectrum between the exact and approximate spectra can be attributed to the interactions among different two-particle configurations which are ignored in the approximate treatment. We also note that the approximate treatment misses the many-body contribution to the spectra at the bottom of the band. Finally, we note that the self-energies agree considerably better than the Green functions This is an important point to keep in mind when basing the treatment of interacting quantum systems on the self-energy. It is well known that even when the self-energy is approximated to be strictly local, the corresponding Green function maintains its global character It is seen that basing an approximate treatment of interacting quantum systems on the Green function rather than the self-energy places much more demanding criteria on agreement with exact results but can also be expected to lead to a more accurate procedure

The last comment provides a strong motivating factor for developing the formalism introduced in this letter. In the case of non-relativistic particles, the Green function of the entire system can be formulated within essentially a single-particle formalism and, since the time-independent Hamiltonian acting on the system as a 
whole is known, (consisting of interparticle interactions), this Green function can be trivially written down. As has been shown, an integration over the coordinates of $n$ particles yields the exact $N-n$-particle Green function, but with its poles shifted by the energies of $n$ single, non-ineracting particles at the $\mathbf{k}=0$ point[8] The corresponding self-energy follows upon inversion of this quantity, and the extreneous shifts in the spectra mentioned above can be removed in a straightforward manner This approach also illustrates in a natural way the possibility of extending the self-energy concept to describe the interaction of $n$ particles with the remaining particles in the system As described above, it also leads to transparent approximate schemes for its evaluation

It is also instructive to consider the effect of the electron density on the singleparticle Green function, shown in Fig 2 The figure shows the single-particle density of states associated with two electrons of opposite spin on a linear ring of four sites (solid line), ten sides (dashed line), and twenty sides (dotted line). The various systems are described by a Hubbard Hamiltonian, with a value of the on-site interaction equal to 10.0. It is clear from these curves that as the number of sites increases, the spectrum approaches that of a single non interacting particle, with the weight of the impurity-like peak at $E \approx 10.0$ becoming progressively weaker. This behavior is to be expected since as the electron density decreases, the likelyhood that two electrons will occupy the same site decreases proportionately. In a later section, we introduce a methodology which is directed at incorporating the presence of a finite density inti the many-particle Green function and hence into the single-particle Green function and self-energy.

\section{CANONICAL MANY-BODY THEORY}

An alternative approach to the calculation of the self energy is provided by the equation of motion of canonical many-body theory, Eq.(1) For the sake of clarity of exposition, we confine our discussion to the two-particle case This is in an important case because the two-particle description of electronic states corresponds exactly to the two-particle nature of the Coulomb interaction. We recall the defition of the t-matrix from Eq.(9), in terms of which the single-particle self-energy is given by the expression[10],

$$
\Sigma\left(x_{4}, x_{2}\right)=\mathrm{i} \int \mathrm{d} x_{3} \mathrm{~d} x_{1} G_{0}\left(x_{1}, x_{3}\right) T^{(2)}\left(x_{1}, x_{2} \mid x_{3}, x_{4}\right) .
$$

This single expression of is taken to include both the direct and the exchange term of the self-energy, through an appropriate definition of the t-matrix in terms of the fully symmetrized (antisymmetrized) free-particle propagator, $G^{(2)}$ It is important to note 
that in energy space, the self-energy can be written as a convolution, its imaginary part, $\Im \Sigma$, taking the form,

$$
\Im \Sigma\left(\mathbf{r}_{4}, \mathbf{r}_{2} ; E\right)=-\mathrm{i} \iint \mathrm{d}^{3} r_{1} \mathrm{~d}^{3} r_{3} \int \mathrm{d} \omega \Im G_{0}\left(\mathbf{r}_{1}, \mathbf{r}_{3} ; \omega\right) \Im T^{(2)}\left(\mathbf{r}_{1}, \mathbf{r}_{2} \mid \mathbf{r}_{3}, \mathbf{r}_{4}, E-\omega\right)
$$

It is also instructive to consider the effect of the electron density on the self-energy and the Green function Figure 3 shows the single-particle Green function obtained directly from the equation of motion, and thus corresponding to a self- energy of the form of Eq.(15) The solid line corresponds to a system of two elections on four sites, and the dashed line to two electrons on ten sites, each system described by a Hubbard Hamiltonian with $U=10.0$ It is interesting to note that, in contrast to Fig. 1, increasing the number of sites, and thus lowering the density, does not affect the structure of the spectra in any significant way This illustrates the fundamental difference between the single-particle Green function obtained in the downfolding procedure discussed above, and the one derived from the equation of motion. The latter contains only those terms of the two-particle Green function which are associated with two electrons occupying the same site and thus feeling the full effect of the Coulomb repulsion The downfolded Green function, on the other hand, contains also contributions from configurations in which the electrons are on different sites and thus not interacting through $U$ Some further discussion of these two different kinds of single-particle Green function is given in the final section We now address the determination of the two-particle t-matrix in a many-particle system, from which the self-energy in either the downfolding or equation-of-motion approach is obtained. This can be done through a consideration of a finite electron density

\section{DFT FOR $N$-PARTICLE STATES}

In order to account for the effects of a finite electron density, especially in infinite systems, we generalize the concept of DFT to the space of $N$ particles

We consider the Hamiltonian of a fully interacting N-particle system,

$$
\hat{H}=\sum_{i}-\nabla_{i}^{2}+\sum_{i} v_{i}+\frac{1}{2} \sum_{i j} v_{i j}
$$

where $\nabla_{i}^{2}$ is the Laplacian operator for particle $\imath, v_{i}=v\left(\mathbf{r}_{i}\right)$ is a single-particle potential for particle $\imath$, and $v_{i j}=v\left(\mathbf{r}_{i}, \mathbf{r}_{j}\right)$ is the interparticle potential, with $v_{i i}=0$ Ordinarily, we are interested in the solutions of the many-particle wave equation,

$$
H \Psi=E \Psi
$$


where $\Psi$ denotes the many-body wave function for the interacting system

We consider the density $n(\mathbf{r})$ for the (non-degenerate) ground state of the system The usual proof of the uniqueness of the external potential as a functional of the density proceeds by reductio ad absurdum Let there be two external potentials, $v(\mathbf{r})$ and $v^{\prime}(\mathbf{r})$, differing by more than a constant and each giving the same ground-state density $n(\mathbf{r})$ Let also $\hat{H}$ and $\hat{H}^{\prime}$ be the corresponding many-body Hamiltonians, and $\Psi$ and $\Psi^{\prime}$ the associated wave functions Both $\hat{H}$ and $\hat{H}^{\prime}$ would correspond to the same ground state density, but the wave functions $\Psi$ and $\Psi^{\prime}$ would be different Take $\Psi^{\prime}$ to be a trial wave function for the system described by $\hat{H}$ (which includes $v(\mathrm{r})$ ) and use the fact that the expectation value of the energy is a minimum for the correct wave function to obtain the result

$$
\begin{aligned}
E_{0}\left\langle\left\langle\Psi^{\prime}|\hat{H}| \Psi^{\prime}\right\rangle\right. & =\left\langle\Psi^{\prime}\left|\hat{H}^{\prime}\right| \Psi^{\prime}\right\rangle+\left\langle\Psi^{\prime}\left|\hat{H}-\hat{H}^{\prime}\right| \Psi^{\prime}\right\rangle \\
& =E_{0}^{\prime}+\int n(\mathbf{r})\left[v(\mathbf{r})-v^{\prime}(\mathbf{r})\right] \mathrm{d}^{3} r
\end{aligned}
$$

where $E_{0}$ and $E_{0}^{\prime}$ denote, respectively, the energy of the $N$-electron system (excluding nuclear-nuclear repulsion), under the influence of $v$ and $v^{\prime}$. The integral $\int n(\mathbf{r}) v(\mathbf{r}) \mathrm{d}^{3} r$ describes the interaction of the charge distribution with the external field. Similarly, taking $\Psi$ as a trial wave function for the system described by $\hat{H}^{\prime}$, we have

$$
\begin{aligned}
E_{0}^{\prime}\left\langle\left\langle\Psi\left|\hat{H}^{\prime}\right| \Psi\right\rangle\right. & =\langle\Psi|\hat{H}| \Psi\rangle+\left\langle\Psi\left|\hat{H}^{\prime}-\hat{H}\right| \Psi\right\rangle \\
& =E_{0}-\int n(\mathbf{r})\left[v(\mathbf{r})-v^{\prime}(\mathbf{r})\right] \mathrm{d}^{3} r
\end{aligned}
$$

Adding the last two equations, we obtain

$$
E_{0}+E_{0}^{\prime}<E_{0}^{\prime}+E_{0}
$$

These inequalities constitute a contradiction to the premise that there can be two different $v$ 's, differing by more than a constant, that give the same density $n(\mathbf{r})$ for the ground state of the system This proves the theorem (the second theorem of Hohenberg and Kohn[16]). We now show that this theorem carries through virtually intact in a hyperspace in which $n$ particles are considered as a single particle We consider explicitly the case $n=2$

Let us consider again the Hamiltonian of Eq (17) but as a sum of distinct, nonoverlapping pairs of particles (so that a given particle belongs to only one pair), labeled by $I$, and write the Hamiltonian in the form,

$$
H=\sum_{I}-\nabla_{I}^{2}+\sum_{I} V_{I}+\frac{1}{2} \sum_{I J} \frac{1}{2} V_{I J}
$$


This Hamiltonian is the same as that above, Eq.(17), except that it is expressed in terms of pairs of particles. We consider explicitly systems with infinite numbers of particles (or with finite but even numbers) so that the partition into pairs can be effected exactly Also, we consider singlet pairs only so that exchange effects on the wave function arise only from the interchange of particles across pairs This partition does not constitute a restriction for zero spin systems Now, we note that for each such pair we can write,

$$
\begin{aligned}
\nabla_{i}^{2}+\nabla_{j}^{2} & =\frac{\partial^{2}}{\partial x_{i}^{2}}+\frac{\partial^{2}}{\partial y_{i}^{2}}+\frac{\partial^{2}}{\partial z_{i}^{2}}+\frac{\partial^{2}}{\partial x_{j}^{2}}+\frac{\partial^{2}}{\partial y_{j}^{2}}+\frac{\partial^{2}}{\partial z_{j}^{2}} \\
& =\sum_{\alpha=1}^{6} \frac{\partial^{2}}{\partial x_{I \alpha}^{2}}=\nabla_{I}^{2}=\nabla_{\mathrm{x}}^{2},
\end{aligned}
$$

where the coordinates of the two particle have been combined in a single coordinate in six dimensions

$$
\begin{aligned}
\mathbf{x} & =\left(x_{i}, y_{i}, z_{i}, x_{j}, y_{j}, z_{j}\right) \\
& =\left(x_{1}, x_{2}, x_{3}, x_{4}, x_{5}, x_{6}\right) .
\end{aligned}
$$

This six-dimensional coordinate forms the direct part of the phase-space (hyperspace) of the two-particles, in which the pair appears as a single particle The other terms that appear in $\mathrm{Eq}(22)$ are uniquely defined in terms of the original Hamiltonian Thus, with $\mathrm{x}=\left(\mathbf{r}_{i}, \mathbf{r}_{j}\right)$, we have,

$$
\begin{aligned}
V_{I} & \equiv V(\mathbf{x})=V\left(\mathbf{r}_{i}, \mathbf{r}_{j}\right) \\
& =v\left(\mathbf{r}_{i}\right)+v\left(\mathbf{r}_{j}\right)+\frac{1}{\left|\mathbf{r}_{i}-\mathbf{r}_{j}\right|},
\end{aligned}
$$

and

$$
\begin{aligned}
V_{I J} & \equiv V\left(\mathbf{x}, \mathbf{x}^{\prime}\right)=V\left(\mathbf{r}_{i}, \mathbf{r}_{j} ; \mathbf{r}_{i}^{\prime}, \mathbf{r}_{j}^{\prime}\right) \\
& =\frac{1}{\left|\mathbf{r}_{i}-\mathbf{r}_{i}^{\prime}\right|}+\frac{1}{\left|\mathbf{r}_{i}-\mathbf{r}_{j}^{\prime}\right|}+\frac{1}{\left|\mathbf{r}_{j}-\mathbf{r}_{i}^{\prime}\right|}+\frac{1}{\left|\mathbf{r}_{j}-\mathbf{r}_{j}^{\prime}\right|}
\end{aligned}
$$

This completely defines the Hamiltonian in Eq (22)

The important feature to notice about this Hamiltonian is that it has a form identical to that in $\mathrm{Eq}$ (17) Therefore, it suggests a treatment using a single-particle framework, where the particles are in six-dimensional (rather than three-dimensional) coordinate space This form of the Hamiltonian allows one to carry through essentially unchanged the proof of the Hohenberg-Kohn theorem so that it holds in the phasespace of two-particles (or $n$ particles) Thus the potential, and hence the energy, is a unique functional of the density, so that we can write for the energy of the electron gas, 


$$
E[n(\mathbf{x})]=T[n(\mathbf{x})]+U[n(\mathbf{x})],
$$

where the various terms are assigned their familiar meaning from ordinary DFT, but must be interpreted as quantities in an appropriate phase space For example, for $n=2$ the term $U[n(\mathrm{x})]$ contains all electrostatic interactions among and between particles in six-dimensional space, as well as their interaction with the nuclei

This generalization of the Hohenberg-Kohn theorem is a straightforward consequence of the observation that the original proof is not dependent on dimensionality. In other words, the theor em holds intact in a configurational space of $3 \mathrm{~N}$ dimensions for systems described by the Hamiltonian of $\mathrm{Eq}(22)$ As is the case with respect to its canonical form, $\mathrm{nDFT}$ yields an energy functional which is stationary with respect to variations in the density and assumes its lowest value for the correct density of the ground state

\section{A The Kohn-Sham equations}

In order to make DFT a usefull tool, explicit expressions for the energy functional $E[n]$ are needed The Kohn-Sham (KS) formalism[11] provides such an expression for ordinary DFT by writing the kinetic energy in terms of single particle states, which can be determined in a straightforward self-consistent scheme, and casting the rest of the kinetic energy as well as the non-local part of the potential energy into the so called exchange and correlation functional, for which suitable approximations such as LDA can be found The KS formalism is reviewed in a number of texts and review articles[12,13] and only some of its more salient features will be mentioned here

We will now attempt to make clear which formal results of the KS formalism hold irrespective of dimensionality and which must be justified when applied to a space with a dimension larger than three

With this in mind, we introduce single-particle orbitals, generalized KS functions, such that

$$
n(\mathrm{x})=\sum_{I}\left|\Psi_{I}(\mathrm{x})\right|^{2}
$$

The meaning of these functions as well as the extent of the sum over $I$ is commented upon below For the moment, we consider these functions as the solutions of an effective "single-particle" Schrödinger equation obtained as follows

We define a single-body potential by the relation,

$$
\begin{aligned}
V(\mathrm{x}) & =\frac{1}{\left|\mathbf{r}_{i}-\mathbf{r}_{j}\right|}+\int \mathrm{d}^{6} x^{\prime} V\left(\mathrm{x}, \mathrm{x}^{\prime}\right) n\left(\mathrm{x}^{\prime}\right) \\
& -\sum_{n} Z_{n}\left[\frac{1}{\left|\mathbf{r}_{i}-\mathbf{R}_{n}\right|}+\frac{1}{\left|\mathbf{r}_{j}-\mathbf{R}_{n}\right|}\right],
\end{aligned}
$$


where $\mathrm{x}=\left(\mathbf{r}_{i}, \mathbf{r}_{j}\right)$, and $V\left(\mathrm{x}, \mathrm{x}^{\prime}\right)$ is given by Eq.(26). For a given density, the energy is evaluated in the usual manner,

$$
\begin{aligned}
E[n(\mathbf{x})] & =T_{s}[n(\mathbf{x})]+\int \mathrm{d}^{6} x V(\mathbf{x}) n(\mathbf{x}) \\
& +J[n(\mathbf{x})]+E_{\mathrm{xc}}[n(\mathbf{x})]
\end{aligned}
$$

where

$$
T_{s}[n(\mathrm{x})]=\sum_{I} \int \mathrm{d}^{6} x \Psi_{I}^{*}(\mathrm{x}) \nabla_{\mathrm{x}} \Psi_{I}(\mathrm{x}),
$$

represents the kinetic energy functional of a non-interacting gas,

$$
J[n(\mathbf{x})]=\frac{1}{2} \int \mathrm{d}^{6} x \int \mathrm{d}^{6} x^{\prime} n(\mathbf{x}) n\left(\mathrm{x}^{\prime}\right) V\left(\mathbf{x}, \mathrm{x}^{\prime}\right),
$$

and $E_{\mathrm{xc}}[n(\mathrm{x})]$ contains the difference between the exact kinetic energy $T[n(\mathrm{x})]$ and that represented by $T_{\mathrm{s}}[n(\mathrm{x})]$, as well as the difference between the exact interparticle interaction and its "classical" approximation[14] given by the expression

$$
\begin{aligned}
E_{\mathrm{xc}}[n(\mathbf{x})] & =T[n(\mathbf{x})]-T_{\mathrm{s}}[n(\mathbf{x})]+\int \mathrm{d}^{6} x_{1} \int \mathrm{d}^{6} x_{2} \\
& \times V\left(\mathbf{x}_{1}, \mathbf{x}_{2}\right)\left[n\left(\mathbf{x}_{1}, \mathbf{x}_{2}\right)-n\left(\mathbf{x}_{1}\right) n\left(\mathbf{x}_{2}\right)\right] .
\end{aligned}
$$

Now, the single-particle Schrödinger equation yielding the solutions $\Psi_{I}(\mathrm{x})$ takes the form,

$$
\left[-\nabla_{\mathrm{x}}^{2}+V(\mathrm{x})+\mu_{\mathrm{xc}}(\mathrm{x})\right] \Psi_{I}(\mathrm{x})=E_{I} \Psi_{I}(\mathrm{x}),
$$

where the exchange-correlation potential is given by the expression,

$$
\mu_{\mathrm{xc}}(\mathrm{x})=\frac{\delta E_{\mathrm{xc}}[n]}{\delta n(\mathrm{x})}
$$

Up to this point, the KS scheme is formally identical to that originally proposed for the case of three-dimensional systems Most importantly, the wave functions (orbitals) $\Psi_{I}(\mathbf{x})$ are to be interpreted only as "aid to calculation", their function being to reproduce the density by means of Eq (28)

The proper implementation of the variatonal principle to the energy functional of Eq (27) is carried out by restricting the set of trial densities to those which are $v$-representable, i e , to densities for which a corresponding local extem nal potential can be found for the interacting Hamiltonian The assumption that underlies the derivation of the KS scheme is that each of these $v$-representable densities can also be represented by a non-interacting Hamiltonian which leads to the single particle 
Schrödinger equation of the form of Eq (34). Such densities are said to be interacting as well as non-interacting $v$-representable. For ordinary particles the assumption is justified when the ground state is non-degenerate [11], but even for degenerate ground states a rigorous extension of the Kohn-Sham theorem can be formulated. This is done by asuming forms for the kinetic energy functional which include a fully antisymetric wave function rather than the product states used for Eq (31) In order to generalize the formalism to $n$-particle states and construct the kinetic energy functional in $n$ particle space, one therefore has to ascertain that the proper symmetry of the wave function with respect to individual partıcles is retained when the system wave function is written in terms of $n$-particle states In the remainder of this section, we will outline how this can be done

We begin by noting that any non-interacting state in conventional three dimensional space can be constructed from a linear combination of Slater determinants Using Laplace's theorem[15] the expanded form of a determinant of order $N$ can be expressed as the sum of products of determinants of lower orders, $n_{i}$, such that in each product $\sum_{i} n_{i}=N$. Furthermore, applied to a Slater determinant, this means that each $n$-order determinant can be associated with the coordinates of a given set of $n$ particles distributed over all possible combinations of $N$ states for the non-interacting system taken $n$ at a time This is consistent with the partition of the Hamiltonian into distinct sets of particles while preserving the symmetry of the wave function required by the exclusion principle.

Now, allow the interaction between particles within each unit to set in which results in an external potential acting on the particles described by the Hamiltonian of Eq.(22). The various determinantal states now evolve under the action of this potential resulting in a system which is "partially" interacting in the context of ordinary particles but strictly non-interacting in terms of $n$-particle units However, the wave function written in terms of the final $n$-particle states, and corresponding to a non-interacting system in hyperspace, leads to a density in that space which is by construction non-interacting $v$-representable in hyperspace Furthermore, this wave function is fully antisymmetric with respect to the coordinates of índividual (three-dimensional) particles.

This discussion has also revealed the extent of the summation over $I$ in Eq.(28) This index runs over all possible combinations of fully interacting $n$-particle states which evolve out of combinations of the states of an $N$-particle system considered $n$ at a time

\section{APPLICATION TO SOLIDS}

The determination of the two-particle Green function and $t$-matrix requires the 
solution of Eq (34). This is a particularly taxing task in the case of an infinite system, such as a solid, and depends crucially on the prior approximate knowledge of the exchange-correlation energy and potential Once an exchange-correlation potential has been determined, (for possible ways of accomplishing this, see below), methods well-established in the treatment of the electronic structure of solids within band theory can be used to obtain the solution One possible way is to generalize the well-known Green function method of Korringa, Kohn, and Rostoker (KKR) to sixdimensional space, as discussed in previous work[9] Such an approach would lead to a two-particle-state representation of the electronic structure The corresponding single-particle quantities, self-energy and Green function can the be obtained from the procedures outlined above (see also discussion in final section)

The determination of the exchange-correlation potential could in principle be carried out in analogy with the determination of that potential in single-particle space. Here, one would treat numerically a collection of six-dimensional particles which interact via the potential of Eq.(26). In this determination, the Coulomb repulsion among the members of any one pair must be turned off. This potential appears as an external field, and its presence would preclude the occurance of a uniform charge density. Also, the intra-pair potential appears explicitly in the Schrödinger equation A numerical representation of the exchange-correlation energy as function of (uniform) two-particle density would then be easily applicable within the local density approximation.

\section{DISCUSSION}

The formalism presented above provides one way for attaining a fairly wide and systematic treatment of correlation effects as they manifest themselves in the calculation of the electronic structure of matter Its basis is not dissimilar from that of recent works $[10,17]$ in the literature in which the LDA or the GW approximation are used as the basis for corrective treatments determining the two-particle t-matrix and hence the single-particle self-energy The most important difference between the present proposal and those works is the suggestion that DFT (and the LDA) can be extended and applied in two-particle space, endowing the entire process with a selfconsistent character In this regard, the methodology is very similar to that proposed by Ziesche[18] and discussed elsewhere in this volume

The use of DFT-LDA in two-particle space depends crucially on the (approximate) knowledge of an exchange-correlation functional One possible method fro determining this functioanl has been suggested in the body of the paper Given such a functional, methods used traditioanlyy in the determination of the electronic structure, such as KKR, can be generalized[9] to obtain the two-particle Green function 
and, hence, the single-particle Green function and self-energy. Because the formalism has revealed two possible procedures for obtaining the single-particle Green function from a higher-order Green function, it is useful to compare and contrast the two processes

An intuitive understanding of the different Green functions can be obtained through a comparison with single-particle Green-functions for defected systems Consider, for example, a binary (possibly disordered) alloy The Green function describing the entire system is given as the trace of the Gieen function operator over the entire space, and it would correspond in a heuristic sense, to the downfolded Green function defined above A partial trace over the space occupied by one of the alloy species yields a partial Green function associated with that species which is analogous to the quantity obtained through the solution of the equation of motion This latter process gives the Green function for particles which are always under the influence of the potential, whereas the downfolding procedure incorporates configurations in $n$-particle space in which the particles do not interact As may be expected, the two types of Green functions coincide in the limit of a full band.

One advantage offered by the present formalism is the possibility of treating a large spectrum of electron-structure realted properties, such as ground-state properties, gaps and satellites, and transport within a unified and systematic framework. Although the method relies on a static potential in two-particle space, it does yield energy-dependent and non-local self-energies, which are in general complex quantities Whether these self-energies provide an accurate treatment of materials properties could be determined through application

\section{ACKNOWLEDGEMENT}

This work was suported by the US Department of Energy through contract No W-7405-ENG-48 with Lawrence Livermore National Laboratory. Research at Oak Ridge was sponsored by the Division of Materials Science, U.S. Department of Energy under contract DE-AC05-96OR22464 with Lockheed Martin Energy Research Corporation. 


\section{REFERENCES}

[1] Alexander L. Fetter and John Dirk Wallecka, Quantum Theory of Many-Partıcle Systems, (McGraw-Hill, New York, 1971)

[2] N H March, W H Young, and S. Sampanthar, The Many-Body Problem in Quantum Mechanics, (Cambridge University Press, Cambridge, 1967)

[3] R M Dreitzler and E K U Gross, Density Functıonal Theory, (Springer-Verlag, Berlin, New York, 1990)

[4] V L Moruzzi, J F Janak, and A R Williams, Calculated Electronıc Properties of Metals, (Pergamon, New York, 1978)

[5] E K U Gross and E. Runge, Vielteilchentheorie, (Teubnes, Stuttgart, 1986)

[6] Tomokazu Kato, Tetsuro Kobayashi, and Mikio Namiki, Supplement of the Progress of Theoretical Physics, (Japan), No 15, (1960)

[7] The DOS shown in the upper panel of the figure was reported earlier in connection with the generalization of DFT to two-particle states in A Gonis, $\mathrm{T}$ C Schulthess, J van Ek, and P E. A Turchi, Phys Rev. Lett 77, 2981 (1996)

[8] The case of a non-uniform but periodic system can be formulated simply through the extension of scalar quantities to matrices

[9] A. Gonis, T. C. Schulthess, P E A Turchi, and J van Ek, Phys Rev B 56, 9335 (1997-I)

[10] M. Springer, F. Aryasetiawan, and K Karlsson, Phys Rev Lett 80, 2389 (1998)

[11] W. Kohn and L. J Sham, Phys Rev 140, A1133 (1965)

[12] R G. Par and Yang, Density Functional Theory of Atoms and Molecules, (Oxford University Press, Oxford, 1989)

[13] R. M Dreitzler and E K. U. Gross, Density Functıonal Theory, (Springer Verlag, Berlin, New York, 1990)

[14] The correlated density, $n\left(\mathbf{r}, \mathbf{r}^{\prime}\right)$ allows the determination of the interparticle electrostatic interaction in the form, $U=\int \mathrm{d}^{3} r \mathrm{~d}^{3} r^{\prime} \frac{n\left(\mathbf{r}, \mathbf{r}^{\prime}\right)}{\left|\mathbf{r}-\mathbf{r}^{\prime}\right|}$.

[15] Thomas Muir, Treatise on the Theory of Determınants, (Dover, New Yolk, 1960)

[16] P Hohenberg and W Kohn, Phys Rev 136, B864 (1964)

[17] C Calandra and F. Mangi, Phys Rev B50, 2061 (1994)

[18] Paul Ziesche, Physics Letters A195, 213 (1994), and this volume 

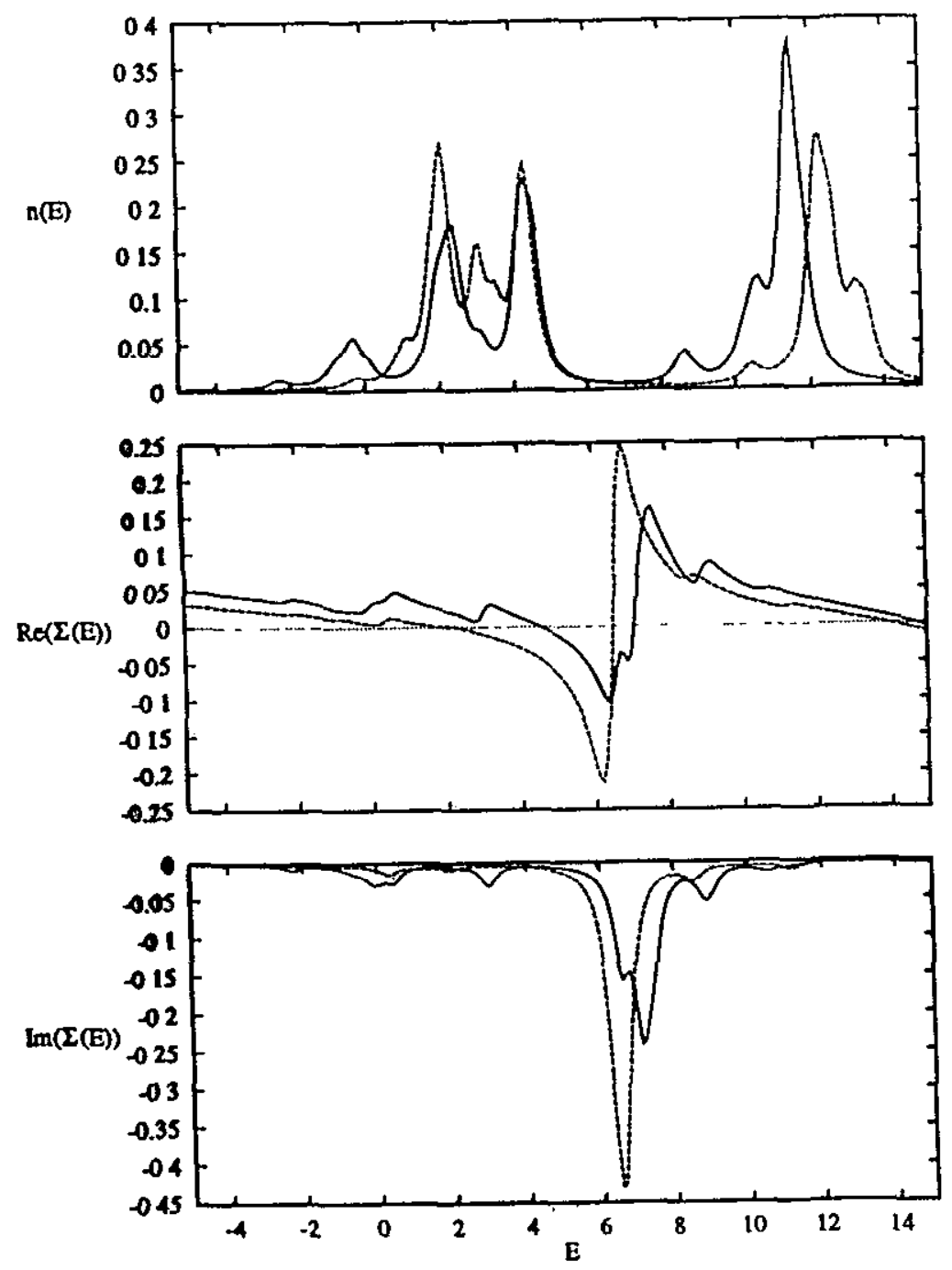


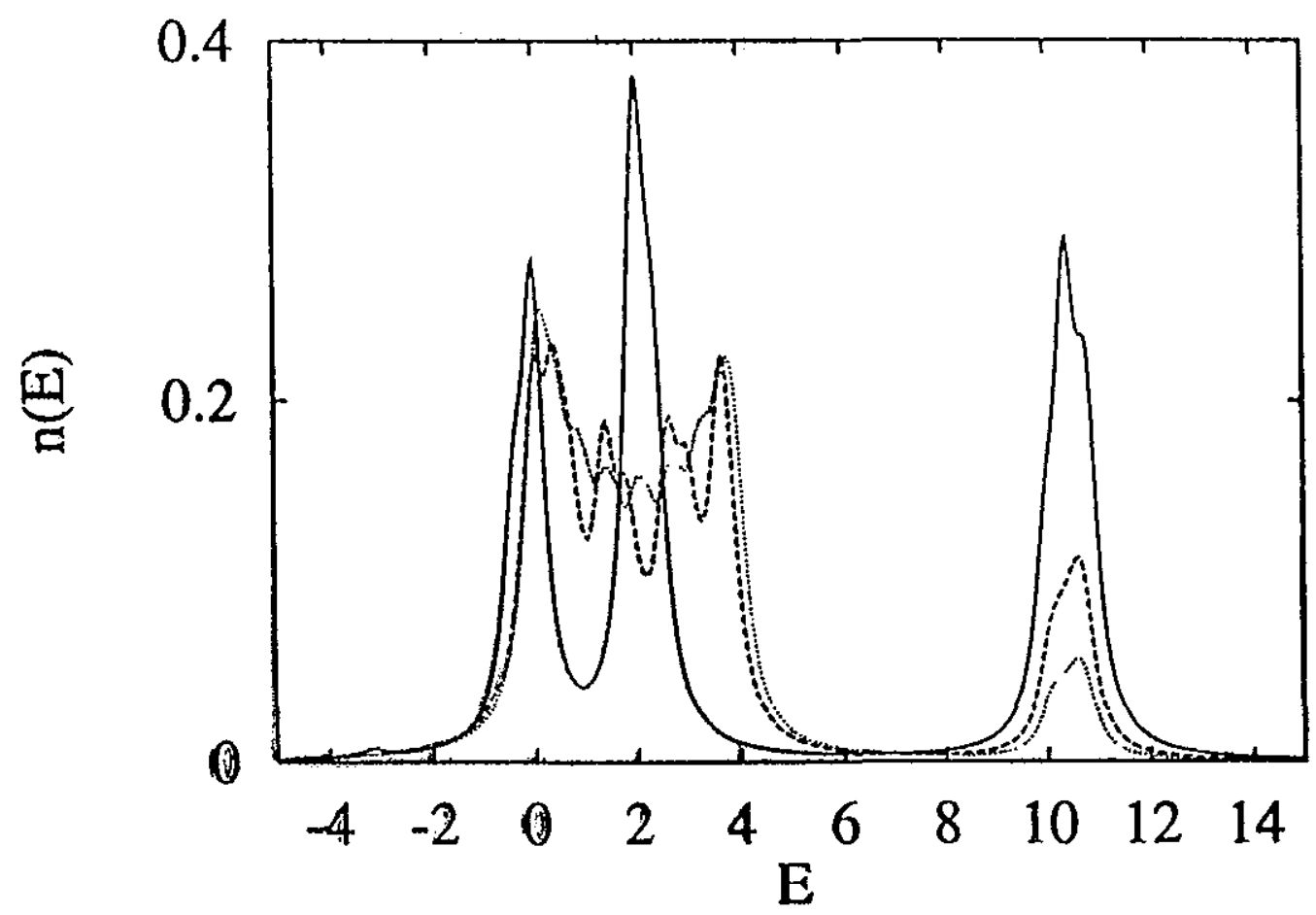




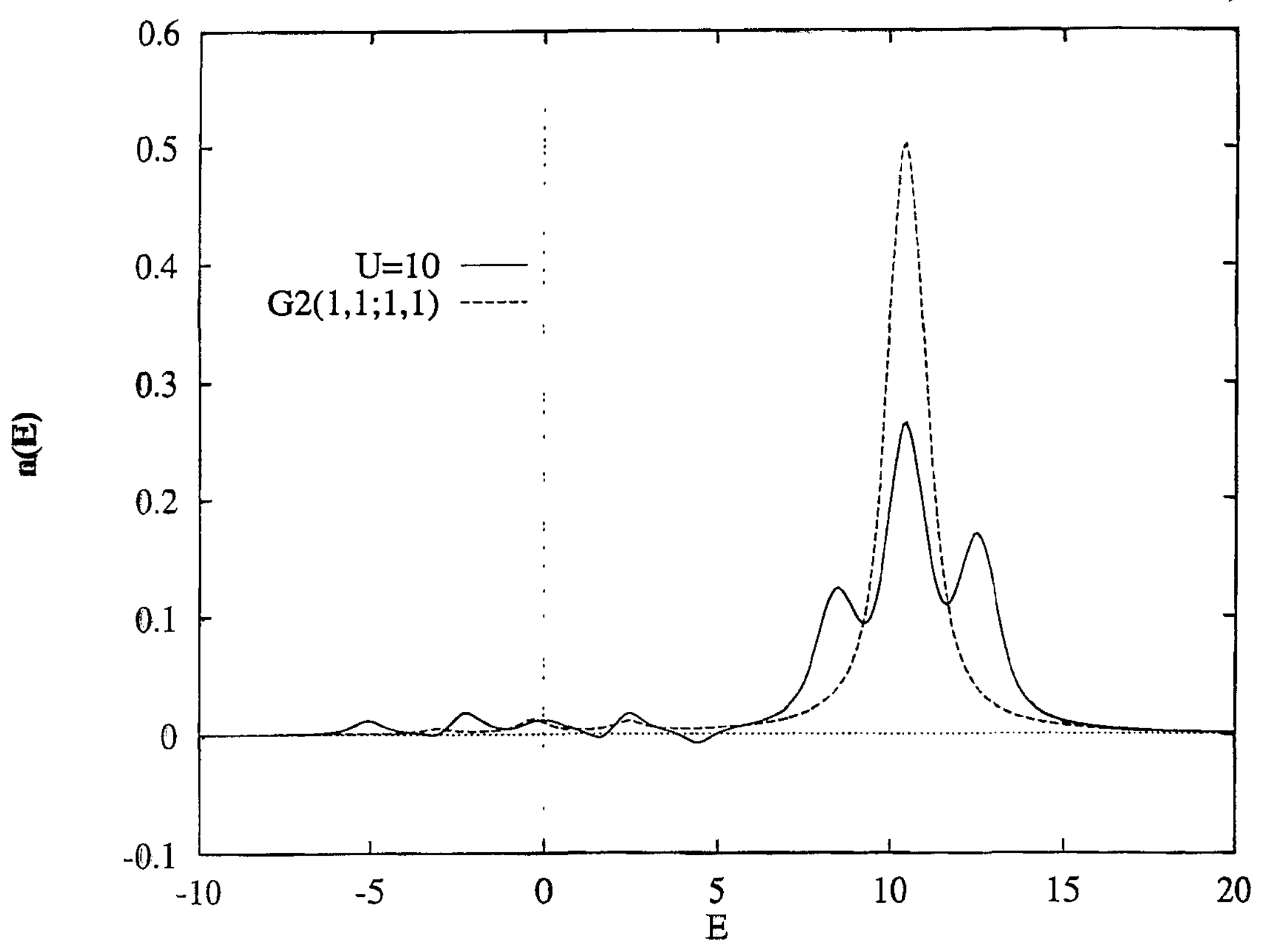

